\title{
ROLE OF METABOLIC SYNDROME AND PRO-INFLAMMATORY STATUS AS POTENTIAL CARDIOVASCULAR RISK FACTORS IN TWENTY TO FIFTY YEARS AGE GROUP IN AN URBAN AREA
}

\author{
Suparna Roy1, Oendri Bhattacharya², Anindya Dasgupta ${ }^{3}$ \\ ${ }^{1}$ Assistant Professor, Department of Biochemistry, Calcutta National Medical College, Kolkata. \\ ${ }^{2}$ Final Year, MBBS Student, Calcutta National Medical College, Kolkata. \\ 3Professor \& HOD, Department of Biochemistry, Calcutta National Medical College, Kolkata.
}

\begin{abstract}
\section{BACKGROUND}

There is a recent trend in increase of cardiovascular mortality in women in their reproductive age group due to several confounding factors including Metabolic Syndrome (MS). Different components used to define MS are reported to differ significantly among different ethnic groups. In the present study, we made an effort to find out the prevalence of MS along with the distribution of their risk factors in male and female subjects in the urban area of our region.
\end{abstract}

\section{METHODS}

100 subjects ( 50 males and 50 females) with heart diseases including acute coronary syndrome, valvular heart disease and cardiac arrhythmia were examined for common anthropometric profile, blood pressure, Fasting Blood Glucose (FBG), lipid profile and hs-CRP. Data were analysed for any difference in the risk factors between male and female subjects and strength of association between different risk factors for MS.

\section{RESULTS}

Student ' $\mathrm{t}$ ' test showed significant high levels of serum triglyceride and hs-CRP in males and serum HDLc, systolic and diastolic $\mathrm{BP}$ in females. On the other hand, the prevalence rate and Odds ratio revealed that among all risk factors in females, the prevalence of high fasting blood glucose $(>110 \mathrm{mg} / \mathrm{dL})$ was highest while the prevalence of raised systolic blood pressure $(>130 \mathrm{mmHg})$ was lowest. However, when the Odds ratio was calculated, it was found to be highest for waist circumference in females (7.79) with serum TG showing the lowest value $(0.724)$. The correlation analysis revealed that waist circumference, not the BMI, was significantly linked to hs-CRP in both the male and female groups.

\section{CONCLUSION}

Among the risk factors for MS, lowered HDLc, higher SBP and DBP and higher FBG values were more prevalent in females while higher hs-CRP and higher triglyceride values were more prevalent in the male group. We conclude that in cardiovascular patients, risk factor for MS are more prevalent in the premenopausal women than their male counterparts.

\section{KEYWORDS}

Cardiovascular Diseases, Metabolic Syndrome, Premenopausal Women, Waist Circumference, Hs-CRP.

HOW TO CITE THIS ARTICLE: Roy S, Bhattacharya O, Dasgupta A. Role of metabolic syndrome and pro-inflammatory status as potential cardiovascular risk factors in twenty to fifty years age group in an urban area. J. Evolution Med. Dent. Sci. 2016;5(71): 51545160, DOI: $10.14260 /$ jemds/2016/1169

\section{INTRODUCTION}

In spite of an effective control over the infection induced mortality around the world after discovery of penicillin in 1940, threats from non-infective disorders like Cardiovascular Diseases (CVD) have been increasing day by day. Along with other developing countries, the situation in India is more alarming. In a study conducted in as early as 1990, it was reported that out of a total 9.4 million deaths in India 2.3 million deaths (25\%) occurred due to cardiovascular diseases. [1] It has been estimated that by the next decade, CVD will be the largest cause of mortality in India with 2.6 million of people succumbing to it.

Financial or Other, Competing Interest: None.

Submission 26-07-2016, Peer Review 20-08-2016,

Acceptance 26-08-2016, Published 02-09-2016.

Corresponding Author:

Dr. Anindya Dasgupta,

Professor \& HOD,

Department of Biochemistry,

Calcutta National Medical College,

32, Gorachand Road, Kolkata-700014.

E-mail: anindya653@gmail.com

DOI: $10.14260 /$ jemds/2016/1169
The Global burden of disease study estimates that the prevalence of CVD deaths in Indians below $70 \mathrm{yrs}$. of age is $54 \%$ against a much lower value of $23 \%$ in the developed countries.[1] It implies a profound adverse effect on the productive manpower and economy of the country.

Conventionally, it was thought that women were less prone to CVD than their male counterparts due to a protective role of oestrogen. ${ }^{[2]}$ But this notion is gradually changing as the rates of death owing to CVD are increasing in young women $(<55$ yrs.) due to several stress factors including those grouped as Metabolic Syndrome (MS). In India, one of the earliest studies undertaken to ascertain the gender specific CVD in 1977-78 reported the incidence of CHD among men and women to be $0.73 \%$ and $0.23 \%$ respectively, ${ }^{[3]}$ whereas later studies carried out in 1987-90 showed a changed incidence of $1.73 \%$ in males and $2.1 \%$ in females.[4] These data clearly indicated that there was a definite trend towards an increased incidence of CVD in women in our country.

Metabolic syndrome known by various names of syndrome $\mathrm{X}$ or insulin resistance syndrome consists of a complex of metabolic abnormalities that may confer increased risk of diabetes mellitus and cardiovascular diseases. In spite of 
several nomenclatures, MS, in brief is defined by a constellation of an interconnected physiological, biochemical, clinical and metabolic factors that directly increase the risk of Atherosclerotic Cardiovascular Disease (ASCVD), Type 2 Diabetes Mellitus (T2DM) and an overall increase in mortality. Generally, the defining criteria for MS include increased Waist Circumference (WC), raised Fasting Blood Glucose (FBG), Insulin Resistance (IR), raised Blood Pressure (BP), dyslipidaemia, pro-inflammatory state and pro-thrombotic state. According to the age and ethnicity of the target population studied, MS has been found to vary worldwide. Among Native Americans, its prevalence was reported to be about $60 \%$ in women aged $45-49$ and $45 \%$ of men aged $45-49$ according to the criteria of the National Cholesterol Education Program and Adult Treatment Panel III.[5] In India, earlier studies reported a prevalence of $30 \%$ and $11 \%$ respectively in the urban areas of Delhi and rural Haryana. However, later studies undertaken yielded highly variable results of MS in different regions of our country. Ramachandran et al[6] reported a prevalence of $41 \%$ in 1995 , while Deepa et al[7] reported a prevalence of $11.2 \%$ in urban Chennai in 1996-97. The sentinel surveillance project reported this prevalence to be $27 \%$ among the industrial population during 2001-03.[8] Components for MS also show significant variations among different groups of population. Prevalence of hypertension showed significant variations between the males and females as reported by several studies in India. Gupta et al (2012) reported a prevalence of hypertension to be $7.6 \%$ and $6.2 \%$ in males and females respectively in the Uttarakhand region, ${ }^{[9]}$ whereas these were reported to be $44 \%$ in men and $45 \%$ in females in Mumbai[5]; $31 \%$ in men and $41 \%$ in females in Trivandrum[10]; $25 \%$ in males and $29 \%$ in females in urban Delhi and $13 \%$ in males and $10 \%$ in females in rural Haryana.[11]

Regarding the prevalence of diabetes and impaired glucose tolerance, a clear trend of increase in incidence has been observed among Indians. In 1972-75, an ICMR conducted large multicentric study reported a $2.6 \%$ and $1.5 \%$ prevalence of diabetes in urban males and females respectively with a lower prevalence of $1.8 \%$ and $1.3 \%$ respectively in rural areas.[12] However, these prevalence rates were found to be substantially increased when ICMR later in 1994 carried out a similar study in people above 20 years of age to find out a prevalence of $14 \%$ among both males and females in the urban areas of Delhi.[11] These data clearly indicate that the prevalence of diabetes is increasing day by day in our country along with a more alarming increase in the females in some areas.

For assessment of the body weight status as a risk factor for MS and cardiovascular risks, several criteria are routinely used like BMI, WC and WHR to measure overall subcutaneous fat distribution, abdominal fat deposit and the ratio between the two respectively. According to the BMI, prevalence of obesity was found to be as high as $48 \%$ in urban area of Delhi and almost similar (26\% and $24 \%$, respectively) among school going boys and girls from affluent society. As in the case of BMI, the prevalence of abdominal obesity as detected by WC varied significantly depending on differing definitions of abdominal obesity. However, the ICMR task force project study reported higher prevalence in the urban Delhi (31\%) and lower in rural Haryana (8\%) using the criteria (WC: $>94 \mathrm{~cm}$ among men and $>88 \mathrm{~cm}$ among women). But with the use of modified ATPIII criteria for the Asians, (WC: $>90 \mathrm{~cm}$ among men and $>85 \mathrm{~cm}$ among women), an overall abdominal obesity of $32 \%$ was reported in Indian industrial population with significant variations between the different parts of the country with Dibrugarh showing the lowest (7\%) and Hyderabad showing the highest (52\%) prevalence.[8] However, when the socioeconomic status among the urban area was concerned, overweight or obesity showed twice prevalence in male subjects in lower income group while almost same prevalence was observed in male and female population of the middle income groups as reflected by the WHR: $\mathrm{M}->0.9, \mathrm{~F}->0.85 .{ }^{[13]}$

High serum cholesterol and low HDLc levels are directly related to cardiovascular diseases, which are significantly influenced by lifestyle transition and urbanisation. Different cross-sectional studies across different parts of the country for the prevalence of hypercholesterolemia reflected variable results like $44 \%$ for males and $50 \%$ for females of Delhi and $37 \%$ for males and $43 \%$ for females in Jaipur, Rajasthan.[14] Importantly, a higher prevalence of hypercholesterolemia was observed in females compared to the males in most of the studies undertaken in India. The atherogenic properties of lowered levels of HDLc and elevated levels of LDLc and VLDLc have made them one of the major criteria for diagnosing MS. High Sensitive C-Reactive Protein (Hs-CRP) is a cytokine biomarker that reflects the low-grade inflammatory state of the body. It has been closely associated with the cardiovascular morbidity and secondary mortality.[15] Association of hs-CRP with BMI has been variable, while that with the abdominal obesity has been more prominent.[16]

All these data indicate that different components used to define MS differ significantly among different ethnic groups with marked variation in distribution of MS in different regions of our country. It prompted us to evaluate the prevalence of this syndrome along with the distribution of their risk factors in male and female subjects in the urban area of our region.

\section{METHODS}

The present study was undertaken as a hospital-based observational study. All patients admitted in the hospital with heart diseases such as acute coronary syndrome, valvular heart disease and cardiac arrhythmia were included following the method of convenience. Depending on the number of patients attending the study centre with CVD, the target sample size was taken to be 100 (one hundred). As obesity increases relative risk of CAD in women by $64 \%$ compared to $46 \%$ in men and women below 50 years old show a two-fold higher mortality rate after an acute MI, the project included fifty male and fifty female subjects in the age group 20 to 50 yrs. from July 2015 to January 2016. The study strictly adhered to the Helsinki declaration for human studies 1975, revised in 2000. Written and informed consent forms have been obtained as per protocol. Institutional Ethical Clearance obtained vide no. CNMC/4, dated 8.7.2015 from the Institution. Following criteria were followed for considering the inclusion and exclusion criteria.

\section{Inclusion Criteria}

All the patients included in the study criteria had a history of ischaemic heart disease, congestive heart diseases, valvular heart disease or cardiac arrhythmias. 


\section{Exclusion Criteria}

Patients with any other pro-inflammatory conditions such as fever, any infective or any inflammatory disorder at present and recent past were excluded from the study to ascertain the significance of elevated hs-CRP levels in the study subjects due to cardiovascular diseases. For the same reason patients with any malignant disorder, neuropsychiatric disorder, thyroid disorder and DM were also excluded. Patients with a history of rheumatic heart disease were excluded from the study, as it is known to put a long-lasting stimulatory effect on the immunological system. Patients with smoking habit and addiction to alcohol or any drugs were also excluded, as all these are known to elevate the levels of hs-CRP to various degrees. For the same reason, patients already on cholesterol lowering drugs were excluded from the study.

\section{STUDY PARAMETERS}

i) Standard Questionnaire: A structured, pre-validated questionnaire containing information on demographics, present and past history and family history of any hypertension or CVD or DM was filled up by taking an appropriate history.

ii) Documentary Evidences for assessing the Disease Severity: Clinical documents and other investigating documents like the ECG report and ECHO reports were obtained to determine the aetiology and severity of the heart disease.

iii) Anthropometric Profile: Body weight in kg and height in metre was determined as per proper protocol.

- BMI was calculated using expression.

- $\quad \mathrm{BMI}=$ Weight $(\mathrm{Kg}) /$ Height $(\text { metre })^{2}$.

- Waist Circumference: WC by a non-expandable measuring tape.

iv) Measurement of BP: Systolic and diastolic blood pressure was measured by calibrated mercury sphygmomanometer. Three readings were taken at supine position and the lowest one was recorded.

\section{v) Biochemical Analysis:}

\section{a) Fasting Blood Sugar (FBG)}

It has been measured by standard Glucose Oxidase Peroxidise (GOD-POD) end point method. Glucose was oxidised to gluconic acid and hydrogen peroxide in presence of glucose oxidase. Hydrogen peroxide further reacted with phenol and 4-aminoantipyrine by the catalytic action of peroxidase to form a red coloured quinoneimine dye complex that was measured against a known standard of glucose solution at 530 $\mathrm{nm}$ in a spectrophotometer. Intensity of colour formed was directly proportional to the amount of glucose present in the sample. Coefficient of Variation (CV) for this assay was 5\% in our laboratory.

\section{b) Serum Triglyceride}

It has been measured by Glycerol 3 Phosphate Oxidase (GPO PAP) end point method. Lipoprotein lipases hydrolysed triglycerides to glycerol and free fatty acids. The glycerol formed reacted with ATP in presence of glycerol kinase to produce glycerol-3-phosphate, which was oxidised by glycerol phosphate oxidase to hydrogen peroxide. The hydrogen peroxide further reacted with phenol and 4-aminoantipyrine by the catalytic action of peroxidase to form a red coloured quinoneimine dye complex that was measured against a known standard of glycerol solution at $530 \mathrm{~nm}$ in a spectrophotometer. Intensity of the colour formed was directly proportional to the amount of triglycerides present in the sample. CV for this assay was $5.7 \%$ in our laboratory.

\section{c) Serum Total Cholesterol}

It has been measured by cholesterol oxidase, phenol and aminophenazone (CHOD PAP). Cholesterol esterase hydrolysis esterified cholesterol to free cholesterol. The free cholesterol is further oxidised to form hydrogen peroxide by the action of peroxidase, which further reacts with phenol and 4aminoantipyrine to form a red coloured quinoneimine dye complex that was measured against a known standard of cholesterol solution at $530 \mathrm{~nm}$ in a spectrophotometer. CV for this assay was $5 \%$ in our laboratory.

\section{d) HDL Cholesterol}

HDLc has been measured by a direct method using Polyethylene Glycol (PEG) CHOD - PAP reagent. In presence of PEG, all the VLDLc and LDLc are precipitated and the HDLc in the supernatant was assayed by cholesterol (CHOP/PAP) reagent. CV for this assay was $6.3 \%$ in our laboratory.

\section{e) Serum LDL Cholesterol}

The assay is based on Polyvinyl Sulfonic Acid (PVS) and Polyethylene Glycol Methyl Ether (PEGME) coupled with classic precipitation method and with improvements in using optimised quantities of PVS/PEGME and selected detergents. LDL, VLDL and chylomicrons react with PVS and PEGME and that results in inaccessibility of LDL, VLDL and chylomicron to cholesterol oxidase and cholesterol esterase, whereas HDL reacts with the enzymes. Addition of R2 containing a specific detergent releases LDL from PVS/PEGME complex. The released LDL reacts with enzymes to produce $\mathrm{H}_{2} \mathrm{O}_{2}$, which is quantified by the Trinder reaction. We found CV for this assay to be $6.8 \%$ in our laboratory.

\section{f) Serum Hs-CRP}

Serum hs-CRP was measured by direct solid phase sandwich ELISA technique. After binding of the hs-CRP with their cognate antibodies fixed on the ELISA microplate, wash procedure was followed to remove unbound excess antigen. The bound antigens reacted with hs-CRP conjugate enzyme and colour was developed by adding TMB substrate. The sample values was calculated from the standard curve prepared by using hs-CRP in the concentration range of 0 , $0.005,0.01,0.025,0.05$ and $0.1 \mathrm{mg} / \mathrm{L}$. The CV for the hs-CRP was $6.5 \%$ in our lab.

\section{g) Quality Control for the Test Parameters}

All analytical methods were calibrated against level 1 and level 2 control materials for each parameter. Control materials were obtained from Lyphochek, BioRad, USA. As mentioned earlier, $\mathrm{CV}$ for all study parameters were below 7 percent.

\section{h) Statistical Analysis}

The statistical analysis were calculated for finding out the differences between the mean values of study parameters between males and females by student ' $t$ ' test, the strength of association between different risk factors by correlation assay and their prevalence by calculating the Odds ratio using SPSS software version number 17 for Windows. 
RESULT AND ANALYSIS

\begin{tabular}{|c|c|c|c|c|c|c|}
\hline & Female & Male & $\begin{array}{l}\text { Prevalence of Risk } \\
\text { Factors in Females }\end{array}$ & $\begin{array}{l}\text { Prevalence of Risk } \\
\text { Factors in Males }\end{array}$ & $\begin{array}{l}\text { Odds Ratio } \\
\text { for Females }\end{array}$ & $\begin{array}{c}\text { Odds Ratio } \\
\text { for Males }\end{array}$ \\
\hline $\begin{array}{c}\text { In risk group } \\
\text { (HDLc }<50 \mathrm{mg} / \mathrm{dL})\end{array}$ & 26 & 21 & 24.5 & 19.8 & $1.75^{*}$ & 0.569 \\
\hline $\begin{array}{l}\text { Not in risk group } \\
(>50 \mathrm{mg} / \mathrm{dL})\end{array}$ & 27 & 32 & & & & \\
\hline $\begin{array}{c}\text { In risk group } \\
\text { (hs-CRP > } 10 \mathrm{mg} / \mathrm{L} \text { ) }\end{array}$ & 27 & 26 & 24.1 & 24.5 & 0.876 & $1.141^{*}$ \\
\hline $\begin{array}{c}\text { Not in risk group } \\
(\mathrm{hsCRP}<10 \mathrm{mg} / \mathrm{L})\end{array}$ & 32 & 27 & & & & \\
\hline $\begin{array}{c}\text { In risk group } \\
(\mathrm{FBG}>100 \mathrm{mg} / \mathrm{dL})\end{array}$ & 45 & 41 & 42.4 & 38.6 & $1.646^{*}$ & 0.6074 \\
\hline $\begin{array}{l}\text { Not in risk group (FBG } \\
<100 \mathrm{mg} / \mathrm{dL})\end{array}$ & 8 & 12 & & & & \\
\hline $\begin{array}{l}\text { In risk group } \\
(\mathrm{WC}>88 \mathrm{~cm})\end{array}$ & 38 & 13 & 35.8 & 12.2 & $7.79 *$ & 0.1282 \\
\hline $\begin{array}{l}\text { Not in risk group } \\
(\mathrm{WC}<88 \mathrm{~cm})\end{array}$ & 15 & 40 & & & & \\
\hline $\begin{array}{c}\text { In risk group } \\
(\mathrm{SBP}>130 \mathrm{mmHg})\end{array}$ & 22 & 7 & 20.7 & 6.6 & $4.663^{*}$ & 0.2144 \\
\hline $\begin{array}{l}\text { Not in risk group (SBP } \\
<130 \mathrm{mmHg})\end{array}$ & 31 & 46 & & & & \\
\hline $\begin{array}{c}\text { In risk group } \\
(\mathrm{DBP}>80 \mathrm{mmHg})\end{array}$ & 23 & 17 & 21.69 & 16.03 & $1.623^{*}$ & 0.6159 \\
\hline $\begin{array}{l}\text { Not in risk group } \\
(\mathrm{DBP}<80 \mathrm{mmHg}\end{array}$ & 30 & 36 & & & & \\
\hline $\begin{array}{c}\text { In risk group } \\
(\mathrm{TG}>150 \mathrm{mg} / \mathrm{dL})\end{array}$ & 31 & 35 & 29.24 & 33.0 & 0.724 & $1.3799 *$ \\
\hline $\begin{array}{l}\text { Not in risk group } \\
(\mathrm{TG}<150 \mathrm{mg} / \mathrm{dL})\end{array}$ & 22 & 18 & & & & \\
\hline $\begin{array}{l}\text { Prevalence of MS in } \\
\text { Percentage** }\end{array}$ & 24 & 10 & 22.6 & 9.4 & 3.55 & 0.281 \\
\hline
\end{tabular}

* Odds ratio more than 1 .

** Metabolic syndrome diagnosed according to the criteria NCEP/ATPIII.

In the above table (Table No. 1), it was noted that among all risk factors in females, the prevalence of high fasting blood glucose $(>110 \mathrm{mg} / \mathrm{dL})$ was highest, while the prevalence of raised systolic blood pressure ( $>130 \mathrm{mmHg}$ ) was lowest. However, when the Odds ratio was calculated, it was found to be highest for waist circumference in females (7.79) with serum TG showing the lowest value (0.724). These data indicate that for similar distribution of FBG, systolic and diastolic blood pressure, reduced HDL and abdominal obesity, the premenopausal female group is more prone to risks for development of CVD with or without MS in our study group. In the above table, it was observed that among all the risk factors of male the prevalence of high fasting blood glucose was highest and that of high SBP is lowest and when Odds ratio were calculated it was found to be highest for hs-CRP in males (1.141) with WC showing the lowest value (0.128).

\begin{tabular}{|c|c|c|c|c|}
\hline Study Parameters & Male (Mean/SD) & Female (Mean/SD) & T Value & P Value \\
\hline TG & $214.2 / 46.9$ & $175.9 / 59.8$ & 3.6 & $<0.001^{*}$ \\
\hline HDLC & $33.0 / 6.4$ & $40.2 / 13.1$ & -3.5 & $0.001^{*}$ \\
\hline SBP & $111.5 / 26.7$ & $125.5 / 17.2$ & -3.15 & $0.002^{*}$ \\
\hline DBP & $71.7 / 13.3$ & $77.6 / 8.3$ & -2.66 & $0.009^{*}$ \\
\hline Hs-CRP & $17.09 / 12.0$ & $12.2 / 10.02$ & 2.23 & $0.027^{*}$ \\
\hline WC & 37.19 .3 .77 & $37.20 / 5.82$ & -0.006 & 0.995 \\
\hline BMI & $24.19 / 4.19$ & $24.42 / 5.12$ & -0.253 & 0.80 \\
\hline FBG & $167.55 / 77.57$ & 0.107 & 0.915 \\
\hline \multicolumn{2}{|c|}{ Table 2: Student 't' Test showing the Differences between the Mean Values of Study Variables }
\end{tabular}

The above table shows that serum TG and hs-CRP were significantly higher in males, while the serum HDLc, SBP and DBP were significantly higher in females. No differences were observed between the WC, BMI and FBG between both sexes. 


\begin{tabular}{|c|c|c|c|c|}
\hline & & \multicolumn{2}{c|}{ Male } & \multicolumn{1}{c|}{ Female } \\
\hline Parameters & $\begin{array}{c}\text { Correlation } \\
\text { Coefficient (r) }\end{array}$ & P Value & 0.266 & P Value \\
\hline R12 & 0.226 & 0.111 & 0.286 & 0.057 \\
\hline R13 & 0.401 & $0.004^{*}$ & 0.202 & $0.040^{*}$ \\
\hline R23 & 0.085 & 0.55 & 0.151 \\
\hline Table 3: Correlation Analysis between Different Study Parameters (1: Waist Circumference, 2: BMI, 3: Hs-CRP)
\end{tabular}

$* \mathrm{P}$ value significant at $\mathrm{P}<0.05$

Correlation study in the Table 3 showed that abdominal obesity as reflected by A was significantly associated with the hs-CRP level in both male and female groups (P value 0.004 and 0.04 respectively for males and females). On the other hand, no such correlation was observed between the whole body obesity indicator BMI and the pro-inflammatory marker hs-CRP ( $P$ value 0.55 and 0.151 for males and females respectively).

\section{DISCUSSION}

In the present study, prevalence of the risk factors was calculated in the male and female population group separately to assess the relative abundance of risk factors between two sexes. Furthermore, different recent studies indicated that the premenopausal women are becoming as prone as their male counterparts. In the above table (Table No. 1), it was noted that among all risk factors in females, the prevalence of high fasting blood glucose was highest, while the prevalence of raised systolic blood pressure was lowest. However, when the Odds ratio was calculated, it was found to be highest for waist circumference in females (7.79) with serum TG showing the lowest risk value $(0.724)$. When the risk values for different risk factors were compared between male and female subjects, Odds ratio was also significantly higher for HDLc, SBP, DBP and FBG in females compared to males. It indicated that impaired glucose tolerance and raised systolic blood pressure are present in higher number of premenopausal females suffering from cardiovascular diseases in our region in comparison to their male counterparts. However, these metabolic derangements were reflected as increased Odds ratio for HDLc, SBP, FBG and WC in the females. Interestingly, there was no significant difference regarding the WC values between the male and female groups, although among the patients suffering from cardiovascular disorders the number of female subjects with WC values more than $85 \mathrm{~cm}$ was significantly higher as indicated by an Odds ratio higher than $1(\mathrm{OR}=7.79$, Table 1$)$. As a serum TG level was not higher in the female group (Table 2 ), the increased abdominal fat deposition does not suggest that it is directly related to the serum TG level. These findings have significant implications regarding the fact that for similar raised values of $\mathrm{TG}$, females have more risk to cardiovascular disorders. Deposition of fat in abdominal tissue, a condition known as visceral obesity is not always related to whole body fat distribution or BMI as found in the present study also (correlation coefficient $r=0.226$ and 0.266 with $p=0.111$ and 0.057 for the males and females respectively). Although, studies showed that in men, WC rather than WHR is the anthropometric index that most uniformly predicts the distribution of adipose tissue in the abdominal region, but it has also been shown that WHR predicts vascular endothelial function in healthy overweight adults and both WC and WHR are predictors of cardiovascular diseases with a significant association of WHR with myocardial infarction risk worldwide.
Regarding the visceral obesity, WC appears as one of the good predictors of Visceral Adipose Tissues (VAT). The relative deposition of fat in the adipose tissues of abdominal visceral adipocytes differs significantly depending on several metabolic, environmental and genetic factors. VAT have been found to directly put an inhibitory effect on adiponectin function more in African American women compared to the African American men.[17] In contrast to the BMI that reflects mainly the subcutaneous fat deposition, abdominal obesity in a greater part reflects visceral fat deposition. Visceral fat deposition has been found to be related to the cardiovascular risks much more prominently than the subcutaneous fat. However, there are some discrepancies to this susceptibility when genetic, racial or gender variations are taken into account. In both white groups and African American population, VAT has been found to be more closely associated with cardiovascular adverse effects and in the African Americans this finding persisted even after adjusting for BMI among the study group. Implications of an increased fat deposition in the abdominal visceral tissues also vary significantly than the overall subcutaneous body fat increase as reflected by an increased BMI. Fat compartments have been shown to be differentially associated with the metabolic risk.[18] Furthermore, variations in fat distribution mediate cardiometabolic risk factors also. Free fatty acids liberated from adipocytes other than the abdominal visceral tissues merge into the systemic circulation and thence reach different tissues throughout the body including the hepatocytes. However, free fatty acids released from the abdominal visceral tissues directly reach the portal circulation and thereby drain into the hepatocytes much earlier in increasing amount. This results in more synthesis of TG in liver that is reflected by an increased VLDLc and LDLc level in the circulation posing more threat for cardiovascular risks. Keeping in track, visceral obesity has been reported to be inversely related to the adiponectin level and its cardioprotective effect.[19] This effect is particularly evident in females, as increased waist circumference in females has a strong mediating effect of insulin resistance in decreasing adiponectin with subcutaneous abdominal obesity in women.[17] All these evidences suggest that VAT provides significant information above and beyond the BMI. Combined with this risk, female population in our study group also showed an Odds ratio of more than one for the cardiovascular risks in them, although the HDL level was not significantly higher in them in comparison to the male subjects as in case of the WC. In spite of that, number of female patients having cardiovascular diseases with an HDL level lower than $40 \mathrm{mg} / \mathrm{dL}$ (The cut-off value of HDL in premenopausal females) is significantly higher when compared to the males as reflected by the Odds ratio (Table 1). It indicates that reduced levels of HDL poses more threat to cardiovascular diseases in premenopausal females in comparison to the males. It implores that in spite of having the protective and positive regulatory function of the oestrogen to keep up the HDL levels in premenopausal women, effects of 
some adverse metabolic factor play major roles in bringing it down that it overall makes the premenopausal women more prone to cardiovascular disease. Low levels of HDL have more predictive value for coronary artery disease in women compared to men.[20] Since HDL is higher in premenopausal women, a modification of the current National Cholesterol Education Program (NCEP) guidelines proposed more aggressive targets for HDL in women.[21] Although, low HDL levels was thought to pose lesser risk for CVD in the absence of elevated LDL cholesterol, total cholesterol or elevated triglyceride levels, recently several studies suggested that an 'isolated low HDL' level represents a significant risk of CHD. ${ }^{[22]}$ The Veterans Affairs Cooperative Studies Program HighDensity Lipoprotein Cholesterol Intervention Trial (VA-HIT) showed that a modest increase in HDL levels in men with CHD and normal LDL levels $(\leq 3.6 \mathrm{mmol} / \mathrm{L})$ resulted in a significant reduction in the risk of major cardiovascular events, but similar data for women are not available so far that can confer the same degree of risk reduction in females. Considering all the pre-existing evidences and the results of the present study, it can be suggested that a gender specific approach in interpreting HDL levels because of the higher absolute levels and possible greater impact of HDL in women. Keeping in track with the metabolic deregulations, both systolic and diastolic blood pressures were found to be significantly higher in females in our study. Greater visceral obesity has been found to have a direct association with hypertension and atherosclerosis. Although suggested initially, but later after correction of all multivariate factors, the BMI was not found to be a significant contributor for development of hypertension[23] and rather WC was found to be the most powerful predictor for development of hypertension, even though the early pre-hypertensive stage. Furthermore, a lower level of adiponectin in visceral obesity promotes the atherosclerotic process that further accentuates the incidence of hypertension in people with greater abdominal fat deposition. Studies have indicated a graded increase in blood pressure with increased abdominal fat even in non-obese population, thus stressing the importance of visceral fat in accentuating systemic blood pressure. It has been suggested that due to the release of hypertensive adipokines like angiotensinogen from the abdominal fat directly into the portal circulation ultimately result in the elevation of systemic blood pressure.

All these results indicate that increased abdominal obesity, even in non-obese people has a potential effect on the aetiology of hypertension and an increase in cardiovascular risk. In our study, the Odds for hypertension as a risk factor was found to be higher in females than the males (4.2 and 1.5 against 0.6 and 0.2 for systolic and diastolic BP respectively), which is in close track with some other studies that too reported a higher prevalence of hypertension in the female population in India.[5,10] The implications of these findings in premenopausal females are particularly important keeping in mind that hypertension is a controllable disease and for each 2 mmHg reduction of blood pressure, 1,51,000 strokes and $1,53,000$ CHD deaths can be prevented throughout the country.[24]

In the present study, the hs-CRP level which indicates the extent of the pro-inflammatory state was found significantly higher in the male subjects suffering from cardiovascular disorders.
Studies have already suggested that a low-grade inflammation is directly associated with the atherosclerosis, coronary heart diseases, metabolic syndrome and defective coagulation processes. The relationship between the cardiovascular disease and elevated hs-CRP level persisted even in the absence of the traditional risk factors.[25] A direct relationship between the systolic blood pressure and the hsCRP level was also found indicating that systolic hypertension is a pro-inflammatory state.[26] In the present study, the correlation analysis indicated a direct straightforward association between the WC and hs-CRP ( $\mathrm{r}=.41$ and $\mathrm{p}=.004$ for males and $r=.28$ and $p=.04$ for females, Table 3). This is in well accordance with other studies that indicated a strong predictive value of WC on low-grade inflammation. Although, the molecular mechanism of visceral adipocyte induced generation of pro-inflammatory state involves an intricate signalling pathway leading to several metabolic, endocrine and immunological functions, mainly hypertrophy of the visceral adipocyte lead to hypoxia-induced damage to the cells leading to release of several cytokines and adipokines in response to the injury.[27] These cytokines and adipokines then play the key role in generating the metabolic, immunological and endocrine abnormalities as reflected by dyslipidaemia, increased hs-CRP level and impaired glucose tolerance with insulin resistance.

With all these observations and analyses, it is evident that the distribution of risk factors for CVD varies between two genders in our study population. Importantly, premenopausal women who were supposed to be resistant to CVD have been found to be equally affected as their male counterparts, if not more in certain circumstances like increased WC, decreased HDL etc. Waist Circumference (WC), which has been found to be a better predictor of diabetes than Body Mass Index (BMI) is now regarded as a reliable indicator of central obesity. The National Cholesterol Education Program Adult Treatment Panel III (NCEP-ATP III) suggested $102 \mathrm{~cm}$ for men and $88 \mathrm{~cm}$ for women as the WC cut-off values for diagnosing MS, but this cut-off point which is based on Western populations is not appropriate for Asians.[28] Asians are more susceptible to obesity-related comorbidities than Caucasians, even when they have smaller WCs or lower BMI values. ${ }^{[29]}$ Hence, keeping in mind the implication of increased WC in Indian population, it is needed to fix the cut-off value of WC to a closer safety margin for Indian population.

\section{CONCLUSION}

It is evident that the confounding factors like hypertension, impaired glucose tolerance, dyslipidaemia, low-grade inflammation and the type of body weight distribution remain similar, but their distribution as risk factors show significant gender variation among the young adults in our study groups. It is a major area for potential intervention and improvement regarding the controlling of these risk factors in gender specific way that will significantly improve scenario and thence the manpower and economic condition of the country.

\section{ACKNOWLEDGEMENT}

The authors acknowledge Indian Council of Medical Research for granting the present research project under STS programme 2015. 


\section{REFERENCES}

1. Murray CJ, Lopez AD. Mortality by cause for eight regions of the world: global burden of disease study. Lancet 1997;349(9061):1269-76.

2. Bush TL, Barrett-Connor E. Noncontraceptive estrogen use and cardiovascular disease. Epidemiol Rev 1985;7: 89-104.

3. Gupta SP, Khetrapaul NK. Incidence of acute myocardial infarction and sudden coronary death in Rohtak city. J Assoc Physicians India 1981;29(5):365-72.

4. Chadha SL, Ramachandran K, Shekhawat S, et al. A 3-year follow-up study of coronary heart disease in Delhi. Bull World Health Organ 1993;71(1):67-72.

5. Gupta PC, Gupta R. Hypertension prevalence and blood pressure trends among 99,589 subjects in Mumbai, India. Indian Heart J 1999;51:691.

6. Ramachandran A, Snehalatha C, Latha E, et al. Rising prevalence of NIDDM in an urban population in India. Diabetologia 1997;40(2):232-7.

7. Deepa R, Shanthirani CS, Premalatha G, et al. Prevalence of insulin resistance syndrome in a selected south Indian population-the Chennai urban population study-7 (CUPS7). Indian J Med Res 2002;115:118-27.

8. Mohan V, Deepa M, Farooq S, et al. Surveillance for risk factors of cardiovascular disease among an industrial population in southern India. Natl Med J India 2008;21(1):8-13.

9. Gupta SK, Dixit S, Singh AK, et al. Prevalence and predictors of hypertension: a cross-sectional study among people coming to a tertiary health care in GarhwalUttarakhand. Indian J Community Health 2012;24(4): 274-9.

10. Joseph A, Kutty VR, Soman CR. High risk for coronary heart disease in Thiruvananthapuram city: a study of serum lipids and other risk factors. Indian Heart J 2000;52(1):29-35.

11. ICMR task force project on collaborative study of coronary heart study in national cardiovascular disease database, 1994.

12. Ahuja MMS. Epidemiological studies on diabetes mellitus in India. In: Ahuja MMS. ed. Epidemiology of diabetes in developing countries. New Delhi: Interprint 1979:29-38.

13. Mohan V, Shanthirani S, Deepa R, et al. Intra-urban differences in the prevalence of the metabolic syndrome in southern India-the Chennai urban population study (CUPS No. 4). Diabet Med 2001;18(4):280-7.

14. Chadha SL, Gopinath N, Shekhawat S. Urban-rural differences in the prevalence of coronary heart disease and its risk factors in Delhi. Bull World Health Organ 1997;75(1):31-8.

15. Sabatine MS, Morrow DA, Jablonski KA, et al. Prognostic significance of the centers for disease control/American heart association high-sensitivity $\mathrm{C}$-reactive protein cut points for cardiovascular and other outcomes in patients with stable coronary artery disease. Circulation 2007;115(12):1528-36.
16. Austin MA. Epidemiology of hypertriglyceridemia and cardiovascular disease. Am J Cardiol 1999;83(9B):13F$16 \mathrm{~F}$.

17. Wei $Q$, Ren $X$, Jiang $Y$, et al. Advanced glycation end products accelerate rat vascular calcification through RAGE/oxidative stress. BMC Cardiovasc Disord 2013;13:13.

18. Fox CS, Massaro JM, Hoffmann U, et al. Abdominal visceral and subcutaneous adipose tissue compartments: association with metabolic risk factors in the Framingham heart study. Circulation 2007;116(1):39-48.

19. Guenther M, James R, Marks J, et al. Adiposity distribution influences circulating adiponectin levels. Transl Res 2014;164(4):270-7.

20. Stensvold I, Urdal $\mathrm{P}$, Thurmer $\mathrm{H}$, et al. High-density lipoprotein cholesterol and coronary, cardiovascular and all cause mortality among middle-aged Norwegian men and women. Eur Heart J 1992;13(9):1155-63.

21. Mosca L, Grundy SM, Judelson D, et al. Guide to preventive cardiology for women. AHA/ACC scientific statement consensus panel statement. Circulation 1999;99(18): 2480-4.

22. Rubins HB, Robins SJ, Collins D, et al. Gemfibrozil for the secondary prevention of coronary heart disease in men with low levels of high-density lipoprotein cholesterol. Veterans affairs high-density lipoprotein cholesterol intervention trial study group. $\mathrm{N}$ Engl J Med 1999;341(6):410-8.

23. Nakanishi N, Nakamura K, Ichikawa S, et al. Risk factors for the development of hypertension: a 6-year longitudinal study of middle-aged Japanese men. J Hypertens 1998;16(6):753-9.

24. Rodgers A, Lawes C, MacMahon S. Reducing the global burden of blood pressure-related cardiovascular disease. J Hypertens Suppl 2000;18(1):S3-S6.

25. Boettger T, Hubner CA, Maier H, et al. Deafness and renal tubular acidosis in mice lacking the $\mathrm{K}-\mathrm{Cl}$ co-transporter Kcc4. Nature 2002;416(6883):874-8.

26. Pitsavos C, Chrysohoou C, Panagiotakos DB, et al. Abdominal obesity and inflammation predicts hypertension among prehypertensive men and women: the ATTICA study. Heart Vessels 2008;23(2):96-103.

27. Emanuela F, Grazia M, Marco de R, et al. Inflammation as a link between obesity and metabolic syndrome. J Nutr Metab 2012;2012:476380.

28. Reaven GM. Banting lecture 1988. Role of insulin resistance in human disease. Diabetes 1988;37(12):1595607.

29. Ferrannini E, Haffner SM, Mitchell BD, et al. Hyperinsulinaemia: the key feature of a cardiovascular and metabolic syndrome. Diabetologia 1991;34(6): 416-22. 